\title{
Binocular Suppression in the Macaque Lateral Geniculate Nucleus Reveals Early Competitive Interactions between the Eyes
}

\author{
(D) Kacie Dougherty, ${ }^{1,2}$ Brock M. Carlson, ${ }^{1}$ - Michele A. Cox, ${ }^{1,3}$ (D) Jacob A. Westerberg, ${ }^{1}$ Wolf Zinke, ${ }^{1}$ \\ (1) Michael C. Schmid, ${ }^{4,6}$ Paul R. Martin, ${ }^{5}$ and ${ }^{\circledR}$ Alexander Maier ${ }^{1}$
}

https://doi.org/10.1523/ENEURO.0364-20.2020

${ }^{1}$ Department of Psychology, College of Arts and Science, Vanderbilt Vision Research Center, Center for Integrative and Cognitive Neuroscience, Vanderbilt University, Nashville, TN 37235, ${ }^{2}$ Princeton Neuroscience Institute, Princeton University, Princeton, NJ 09540, ${ }^{3}$ Department of Brain and Cognitive Sciences, University of Rochester, Rochester, NY 14620, ${ }^{4}$ Faculty of Science and Medicine, University of Fribourg, Fribourg $\mathrm{CH}-1700$, Switzerland, ${ }^{5}$ Save Sight Institute and Australian Research Council Centre of Excellence for Integrative Brain Function, The University of Sydney, Sydney, New South Wales 2006, Australia, and ${ }^{6}$ Biosciences Institute, Faculty of Medical Sciences, Newcastle University, NE2 4HH, UK

\begin{abstract}
The lateral geniculate nucleus (LGN) of the dorsal thalamus is the primary recipient of the two eyes' outputs. Most LGN neurons are monocular in that they are activated by visual stimulation through only one (dominant) eye. However, there are both intrinsic connections and inputs from binocular structures to the LGN that could provide these neurons with signals originating from the other (non-dominant) eye. Indeed, previous work introducing luminance differences across the eyes or using a single-contrast stimulus showed binocular modulation for single unit activity in anesthetized macaques and multiunit activity in awake macaques. Here, we sought to determine the influence of contrast viewed by both the non-dominant and dominant eyes on LGN single-unit responses in awake macaques. To do this, we adjusted each eye's signal strength by independently varying the contrast of stimuli presented to the two eyes. Specifically, we recorded LGN single unit spiking activity in two awake macaques while they viewed drifting gratings of varying contrast. We found that LGN neurons of all types [parvocellular (P), magnocellular $(\mathrm{M})$, and koniocellular $(\mathrm{K})$ ] were significantly suppressed when stimuli were presented at low contrast to the dominant eye and at high contrast to the non-dominant eye. Further, the inputs of the two eyes showed antagonistic interaction, whereby the magnitude of binocular suppression diminished with high contrast in the dominant eye, or low contrast in the non-dominant eye. These results suggest that the LGN represents a site of precortical binocular processing involved in resolving discrepant contrast differences between the eyes.
\end{abstract}

Key words: binocular; interocular suppression; lateral geniculate nucleus; macaque; monocular; vision

\section{Significance Statement}

A fundamental feature of the primate visual system is its binocular arrangement, which affords stereovision and hyperacuity. A consequence of this arrangement is that the two eyes' views need to be resolved to yield singular vision, which is normally accomplished by fusion or suppression of one of the eye's inputs. This binocular processing has been shown to occur in cortex, subsequent to thalamic processing. Here, we show that neurons in the lateral geniculate nucleus (LGN) receiving excitatory retinal input from one eye can be suppressed by high-contrast visual stimulation of the other eye, indicating that the geniculate serves as a precortical site of binocular processing.

Received August 20, 2020; accepted November 28, 2020; First published January 25, 2021.

The authors declare no competing financial interests.
Author contributions: K.D., M.A.C., M.C.S., and A.M. designed research; K.D., B.M.C., J.A.W., and W.Z. performed research; P.R.M. contributed unpublished reagents/analytic tools; K.D., W.Z., and P.R.M. analyzed data; K.D. and A.M. wrote the paper. 


\section{Introduction}

The lateral geniculate nucleus (LGN) is the main recipient of the outputs of the two eyes in primates. Its anatomic organization raises the question of the role it plays in resolving binocular inputs to support singular vision. In diurnal primates, the LGN comprises distinct layers and virtually all neurons within each layer receive direct inputs from only one eye (Kaas et al., 1972). Neurons within a layer receive input from one eye and neighboring layers contain neurons that receive input from the other eye. Congruent with their inputs, almost all LGN neurons are monocular, exclusively excited by stimulation of one eye. Nevertheless, LGN neurons might interact across layers (Campos-Ortega et al., 1968; Saini and Garey, 1981) or receive inputs from structures with binocular neurons (Hubel and Wiesel, 1968; Lund et al., 1975). The effects of these influences could manifest as a difference in magnitude of visual responses under binocular stimulation relative to monocular stimulation.

Studies in cats have well established that spike rates of most LGN neurons are altered by binocular stimulation (Sanderson et al., 1971; Schmielau and Singer, 1977; Xue et al., 1987; Tong et al., 1992; Sengpiel et al., 1995). Binocular modulation also occurs in the primate. Unlike in the cat, related work in macaques has not focused on visual contrast. Instead, previous work on binocular modulation either introduced luminance differences across the eyes (Rodieck and Dreher, 1979; Schroeder et al., 1990) or introduced stimuli at one contrast level (Marrocco and McClurkin, 1979). One reason to address this question in macaques is that cat LGN differs anatomically from primate LGN (for review, see Dougherty et al., 2019). Assessing binocular modulation in the LGN as a function of visual contrast in both eyes would reveal how interactions between the eyes are influenced by the strength of each eye's signal. Contrast-dependent binocular interactions in the LGN could have implications for known psychophysical phenomena, such as interocular suppression.

The primate LGN is composed of three major cell classes, parvocellular (P), magnocellular (M), and koniocellular $(\mathrm{K})$ neurons, with known functional and anatomic distinctions. Known differences in contrast sensitivity among these parallel pathways (Shapley et al., 1981; Derrington and Lennie, 1984; Norton et al., 1988; Lee et

This work was supported by the National Eye Institute Grant 1R01EY027402-03 and the National Eye Institute Training Grant 5T32EY007135-23 (to J.A.W. and K.D.), the National Institute of Mental Health Training Grant 5T32MH065214-17 (to K.D.), and National Eye Institute Training Grant 1F31EY031293-22 (to J.A.W.). M.C.S. is supported by the European Research Council Grant OptoVision 637638.

Acknowledgements: We thank M. Schall, B. Mitchell, L. Daumail, M. Feurtado, C. Jones, K. Shuster, M. Maddox, S. Motorny, P. Henry, D. Richardson, K. Torab, C. Subraveti, M. Johnson, B. Williams, and R. Williams for technical advice and assistance. We also thank B. Cumming, V. Casagrande, Y. Jiang, and F. Briggs for input and feedback at an early stage of this work.

Correspondence should be addressed to Kacie Dougherty at kacied@ princeton.edu.

https://doi.org/10.1523/ENEURO.0364-20.2020

Copyright (C) 2021 Dougherty et al.

This is an open-access article distributed under the terms of the Creative Commons Attribution 4.0 International license, which permits unrestricted use, distribution and reproduction in any medium provided that the original work is properly attributed. al., 1989a; Sclar et al., 1990) as well as their physical distribution could impact whether contrast-dependent binocular interactions occurs for these groups. Based on the studies that assessed general binocular modulation, it remains unclear whether this modulation occurs only for $M$ or both P and M neurons (Marrocco and McClurkin, 1979; Rodieck and Dreher, 1979). Furthermore, whether K neurons, intercalated between different eye-dominant layers, show binocular modulation in macaques is an outstanding question. Recent work in anesthetized marmosets, focusing on $\mathrm{K}$ layers, found that about one third of $\mathrm{K}$ neurons get excitatory binocular input (Zeater et al., 2015), and that most $\mathrm{K}$ neurons with excitatory responses to high-contrast stimuli were suppressed by binocular stimulation (Belluccini et al., 2019). A second question is how binocular modulation of single neurons may be impacted by anesthesia, as anesthesia is known to affect binocular processing in this structure (Schroeder et al., 1988). Only one study thus far has considered LGN binocular processing in the awake state (Schroeder et al., 1990). However, these measures were based on population spiking, which could have included spikes from neighboring eye dominate layers.

We sought to determine how visual contrast viewed by both eyes impacts the visual response of LGN neurons receiving direct input from one eye only in awake primates. To do this, we varied the contrast of stimuli shown to each eye independently to adjust the signal strength each eye carries. Two macaques viewed drifting sine-wave gratings presented to one or both eyes while spiking of LGN neurons was recorded with a linear multicontact electrode array. We found that significant binocular modulation occurred when a high contrast stimulus drove the non-dominant eye while a low contrast stimulus was presented to the dominant eye. Individual LGN units showing this pattern comprised $\mathrm{P}, \mathrm{M}$, and $\mathrm{K}$ groups. This result suggests that inhibitory input from the non-dominant eye can suppress LGN neurons in awake primates only if the dominant eye input is too weak to overcome this inhibition. In other words, there is a weak, antagonistic relationship between the two eyes' signals within primate LGN that only becomes evident at certain interocular contrast differences. This finding resolves earlier discrepant findings as to types of neurons showing binocular modulation, and suggests that the LGN is a precortical binocular processing site that may initiate the process of resolving discrepant contrast differences across the eyes.

\section{Materials and Methods}

Two adult monkeys (Macaca radiata, one male) were used in this study. All procedures followed regulations by the Association for the Assessment and Accreditation of Laboratory Animal Care (AAALAC), the University's Institutional Animal Care and Use Committee (IACUC) and National Institutes of Health (NIH) guidelines. Each monkey received nutritionally complete food biscuits, quantity based on recommendation by the veterinarian, in the morning and afternoon every day. In addition, animals were provided fresh produce and other forms of environmental enrichment on five or more days a week. The animals were water regulated during the weeks that the 
experiments were conducted. They were trained to fixate and received juice reward following completed trials.

\section{Surgical procedures}

Before experiments began, each monkey underwent two separate surgeries conducted under sterile conditions. In the first surgery, the animals were implanted with a custom-designed plastic head holder. In the second surgery, a craniotomy was made and a plastic recording chamber (Crist Instruments) was implanted. The craniotomy was centered on stereotaxic coordinates above the LGN (anterior-posterior: $7 \mathrm{~mm}$, medial-lateral: $12 \mathrm{~mm}$ ). The head holder and the recording chamber were attached to the skull using either transcranial ceramic screws or titanium screws (Thomas Recording) and self-curing dental acrylic (Lang Dental Manufacturing). Animals were administered isoflurane anesthesia (1.5-2.0\%), and vital signs, such as blood pressure, heart rate, $\mathrm{SpO}_{2}, \mathrm{CO}_{2}$, respiratory rate and body temperature were continuously monitored throughout both surgeries. Following surgery, each monkey was administered analgesics (buprenorphine) and antibiotics (ceftiofur sodium, unless otherwise directed by the veterinarian) by intramuscular injection. Researchers, facility veterinarians, and animal care staff closely observed the animals for at least $3 \mathrm{~d}$ following surgery.

\section{Visual display}

Stimuli were presented on a linearized cathode-ray tube monitor with a refresh rate of either $60 \mathrm{~Hz}$ with resolution $1280 \times 1024$ (for 1 unit) or $85 \mathrm{~Hz}$ with resolution $1024 \times 768$ (for 50 units), or on a linearized LED (VPixx) display with a refresh rate of $120 \mathrm{~Hz}$ with resolution $1920 \times 1080$ (for 15 units). Visual stimuli were generated using custom-written code for MonkeyLogic (Asaad et al., 2013) in MATLAB (R2012-2014; The MathWorks) on a PC operating Windows 7 or Windows 10 with an NVIDIA graphics card. The stimuli were viewed through a calibrated stereoscope consisting of infrared (IR)-light passing cold mirrors (Edmund Optics; Fig. 1A). The experimental setup was configured so that the animal's right eye viewed stimuli presented on the right side of the monitor and the animal's left eye viewed stimuli on the left side of the monitor. A black, non-reflective septum was placed between the monitor and the back side of the mirrors, effectively dividing the left and right sides of the apparatus, to prevent light scatter from one side of the monitor to the opposing eye.

IR light-sensitive cameras, placed directly behind the cold mirrors on the stereoscope, were used to track gaze position with commercially available eye tracking software (Eye Link II, SR Research). Both eyes were tracked using two separate cameras in the majority of recording sessions. Gaze position was converted to an analog signal and inputted to MonkeyLogic/MATLAB (NIDAQ PCl$6229)$ at $1 \mathrm{kHz}$. The stereoscope was calibrated to facilitate binocular fusion of the left and right sides of the monitor using a behavioral task that relied on acquiring the same gaze position for corresponding locations on each side of the monitor at the beginning of each recording session. In this task, the animal was required to move
A
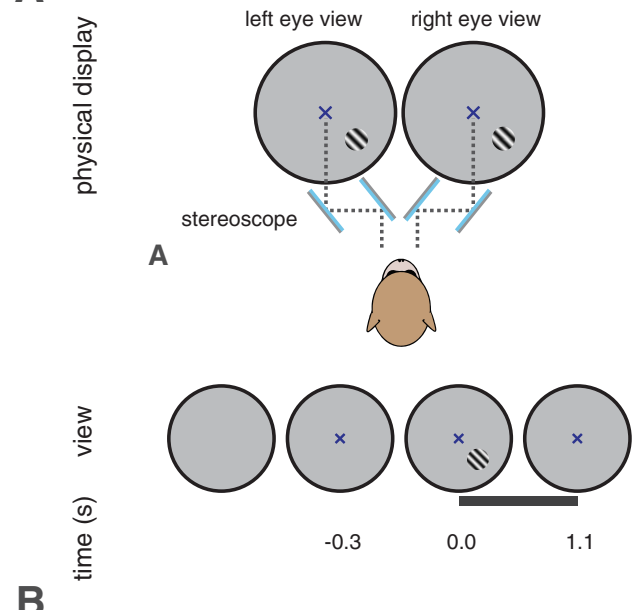

B

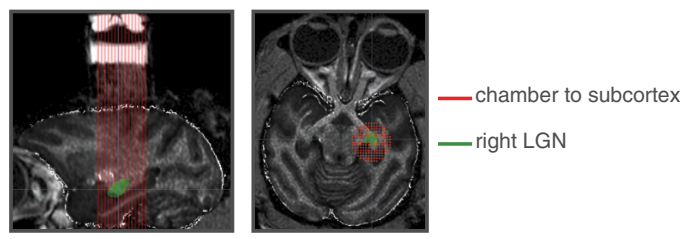

C

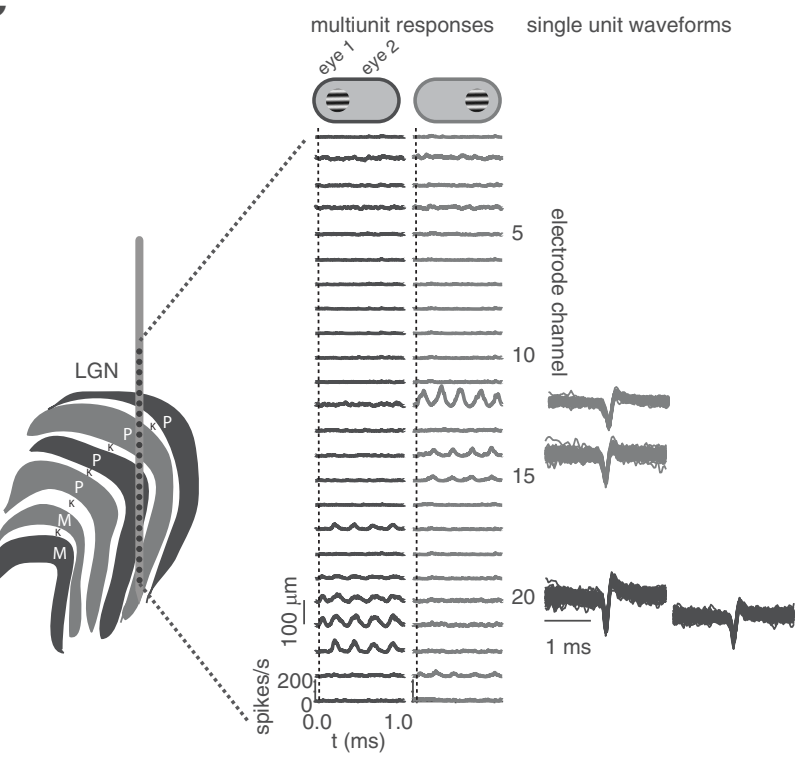

Figure 1. Experimental setup. A, Visual stimuli were presented through a mirror stereoscope consisting of two pairs of mirrors angled so that the subjects fused the left and right sides of the display. Animals fixated a central cross that was presented dioptically before a monocularly or binocularly presented drifting sine-wave grating appeared over the receptive field of the recorded neurons for $1000-1100 \mathrm{~ms}$. Schematic not shown to scale. B, Sagittal (left) and axial (right) MRI of monkey I34 used to guide electrode placement within the well placed over the right hemisphere's LGN. C, To-scale schematic of linear multicontact electrode array in the LGN (left). In the example session shown, multiunit activity on nine contacts exhibited monocular, linear visual responses to the drifting grating, with eye dominance changing across presumed layer boundaries. Some, but not all, of these spikes were classified as single units (right). See Materials and Methods for details. 
their gaze position to a single fixation cue that was placed at the center of the left side of the monitor, shown to the left eye only, and gaze position was recorded. The single fixation cue was moved to eight other locations one-at-atime, spaced equidistant from the left monitor's center up to $10^{\circ}$. Gaze position was recorded for each cue location. This procedure was repeated for the right side of the monitor. Then, gaze positions for corresponding cue locations from the left and right sides of the monitor were compared. Overlapping gaze positions for corresponding cue locations suggest the mirrors are optically aligned for binocular fusion. We confirmed that binocular fusion could occur by showing a central fixation cue to the left eye and right eye in alternation, monitoring for any change in eye position. An oval aperture or set of intersecting circles in each corner was displayed at the edge of each halfscreen to further aid fusion.

Cone-isolating stimuli were generated by convolving the monitor $(R, G, B)$ channel spectral power distribution with absorbance templates (Lamb, 1995) for cones with peak sensitivity in short-wavelength $(S ; 420 \mathrm{~nm})$, mediumwavelength $(\mathrm{M} ; 530 \mathrm{~nm})$, or long-wavelength $(\mathrm{L} ; 558 \mathrm{~nm})$ bands of the visible spectrum. Monitor spectral power distributions were measured using a PR-655 photometer (Photograph Research). Cone templates were adjusted to account for lens absorption and pigment self-screening assuming axial absorbance of 0.15 and outer segment length $20 \mathrm{~nm}$. No correction for macular pigment was incorporated because the vast majority of receptive field eccentricities were above $2^{\circ}$.

\section{Neurophysiological recordings and data acquisition}

Following the second surgery, the location of the LGN was mapped by sampling different locations of a grid with $1 \mathrm{~mm}$ spacing (Crist Instruments) that was placed inside the chamber (Fig. 1B). Specifically, a linear multicontact array (V-Probe, U-Probe, Plexon Inc.) with either 24 or 32 contacts of $0.1-\mathrm{mm}$ intercontact spacing was lowered through a guide tube placed within the chosen grid hole. After the probe tip left the guide tube, it was moved at rate of $\sim 10 \mu \mathrm{m} / \mathrm{s}$ to reach the LGN. Each encountered receptive field was first manually mapped using drifting sinewave gratings, Gabor-filtered sine wave gratings or rectangular bars of high contrast and luminance. These stimuli were moved on the display while the animal fixated a central cue. The stimulus was then placed in corresponding RF locations in both eyes to verify that excitatory responses were monocularly driven. The geometry of the LGN and angle of approach meant that sometimes spiking on several electrode contacts could be stimulated by the same stimulus ( $\sim 2.5-5^{\circ}$ diameter), but this was not always the case (Fig. 1C). In all, 29 sessions of LGN data were recorded with a linear multicontact array, and 11 sessions were recorded with a standard single-contact tungsten microelectrode (FHC Inc).

During each session, extracellular voltage fluctuations (filtered at $0.5 \mathrm{~Hz}$ to $30 \mathrm{kHz}$ ) were recorded inside an electromagnetic-shielded booth. These signals were amplified, filtered and digitized using a 128-channel Cerebus Neural Signal Processing System (NSP; Blackrock Microsystems).
A bandpass filtered $(0.5 \mathrm{~Hz}$ to $7.5 \mathrm{kHz})$ signal sampled at $30 \mathrm{kHz}$ was saved for offline analysis. The NSP system also digitized the analog output of an IR-based eye tracking system (EyeLink II, SR Research) and the output of a photodiode (OSI Optoelectronics) that was placed on the monitor to timestamp stimulus-related events. The NSP also recorded digital event codes that were sent from the behavioral control system (MonkeyLogic; Asaad et al., 2013). The photodiode signal and event markers were used to align the neural data with visual and behavioral events.

All neurophysiological signals were extracted offline using custom written code in MATLAB (2016a and 2019a; The MathWorks). Single neurons were extracted with KiloSort (Pachitariu et al., 2016), using default parameters for sorting and cluster merging, with a few exceptions such as changing the threshold to detect spikes to 2.5 SDs. For all multicontact electrode sessions, the results of KiloSort were viewed in Phy, an open source analysis package (https://github.com/cortex-lab/phy). Using Phy, clusters were manually split or merged when appropriate, and were rated for isolation quality. The channel on the probe on which the cluster was recorded was determined using code from the University College London Cortex Lab (https://github.com/cortex-lab/spikes).

Spike rates were downsampled to $1 \mathrm{kHz}$. For each neuron, spike times were converted to a time-varying signal using 0 to represent time points without a spike and 1 for time points where a spike was detected. The time-varying signal was then convolved using a Poisson distribution resembling a postsynaptic potential (Sayer et al., 1990), with the spike rate $(R)$ computed at time $(t)$ :

$$
R(t)=\left[1-\exp \left(-\frac{t}{\tau_{g}}\right)\right] *\left[\exp \left(-\frac{t}{\tau_{d}}\right)\right],
$$

where $\tau_{g}$ and $\tau_{d}$ are the time constants for growth and decay, respectively. Values of 1 and 20 for $\tau_{g}$ and $\tau_{d}$ respectively were used based on previous studies (Hanes et al., 1995). After convolution, units were converted to spikes per second by multiplying the signal by the sampling frequency.

\section{Experimental design and statistical analysis}

During each session, data were collected while the animal viewed drifting sine-wave gratings of varying contrast presented to one or both eyes (this paradigm is hereinafter referred to as the binocular contrast paradigm). The animal was required to fixate within a $1-2^{\circ}$ radius around the central fixation cue $\left(0.5^{\circ}\right)$. All gratings were presented at temporal frequency of $4 \mathrm{~Hz}$ (except for one session at $8 \mathrm{~Hz}$ ) and spatial frequency of $1 \mathrm{cycle} /{ }^{\circ}$. Within each recording epoch, gratings were presented at constant orientation ( $N=27$ units horizontal, $N=19$ vertical, $N=10$ non-cardinal).

In addition to the binocular contrast paradigm, we collected data to identify whether neurons belonged to the $P$, $\mathrm{M}$, or $\mathrm{K}$ subclass of LGN neurons. For 27 sessions, we collected spiking data while we presented cone-isolating stimuli designed to modulate along the $L^{+} M^{+} / L^{-} M^{-}$, $\mathrm{L}^{+} \mathrm{M}^{-} / \mathrm{L}^{-} \mathrm{M}^{+}$, or $\mathrm{S}^{+} / \mathrm{S}^{-}$axes in $\mathrm{DKL}$ color space (Derrington 
et al., 1984; Lee et al., 1989a). In each trial, the animal fixated while a cone-isolating stimulus with temporal frequency of $4 \mathrm{~Hz}$ and spatial frequency of 1 cycle degree ${ }^{-1}$ drifted over the RF location of either the dominant eye, or both eyes simultaneously for $\sim 1000-1100 \mathrm{~ms}$. For 12 other sessions, we collected spiking data while we presented alternating (red-to-green or blue-to-yellow) color patches to the receptive field while the animal fixated. We used converging evidence, including the shape of the contrast response curves, the responses to the spectral stimuli, and contextual information from multicontact arrays (chiefly, recording depth and the character of background multiunit activity) to label each unit as part of the $\mathrm{P}, \mathrm{M}$, or K functional streams (Wiesel and Hubel, 1966; Shapley et al., 1981; Derrington et al., 1984; Derrington and Lennie, 1984; Norton et al., 1988; Lee et al., 1989a; Sclar et al., 1990; White et al., 1998, 2001). If the overall spike density function across a single file with the binocular contrast paradigm had a mean of $<1.9$ spikes/s, we excluded the cluster from further analysis. We also excluded units that had ambiguous or atypical contrast response functions (CRFs).

To assess the stability of the units during the binocular contrast paradigm, we summed the spike counts of the time-varying, trial-aligned spiking data for the period $600 \mathrm{~ms}$ before stimulus onset through $1300 \mathrm{~ms}$ following stimulus onset. We computed a running average (movmean.m from MATLAB, with window length equal to $20 \mathrm{~ms}$ ) of these counts across trials, then identified likely points of instability in the mean spike rate (findchangepts. $m$ from MATLAB). We removed periods of instability using this algorithm. We checked the results of the algorithm for each unit. In rare cases, we manually removed periods of instability based on judicious visual inspection.

Neurons were further analyzed whether they were recorded for at least 12 trials for monocular and binocular conditions, each with 0.5 contrast or higher. For each unit and for every trial, we computed the autocorrelation on the time-varying, trial-aligned spiking data for the period between 0 and $1100 \mathrm{~ms}$ relative to stimulus onset. We normalized the autocorrelation for each trial by dividing by the total number of spikes in that trial. We then calculated the power spectral density (PSD) of the autocorrelations using a method designed to extract oscillatory components from 1/f-trending data (Wen and Liu, 2016). We refer to the PSD for the oscillatory (rhythmic) components as the oscillatory PSD, and for the (arrhythmic) 1/f components as the fractal PSD. Recorded units were considered to show significant visual response if two requirements were met: (1) power of the oscillatory PSD at the grating drift frequency exceeded the power of the fractal PSD at that same frequency for blank (no stimulus) trials based on a one-tailed $t$ test ( $\alpha=0.05)$; and (2) the power of the oscillatory PSD at the grating drift frequency exceeded the power of the fractal PSD at the same frequency based on a one-tailed $t$ test $(\alpha=0.05)$.

Responses to visual stimuli were based on the power of the oscillatory PSD at the grating drift frequency. Binocular modulation was assessed by the percent difference between the monocular response and the binocular response for each unit. Outliers (values that were more than three scaled median absolute deviations from the median) were excluded from both the figures and statistical analyses. Distributions of binocular modulation were fit with a non-parametric kernel smoothing function, with a normal distribution shape for the component curves, in MATLAB.

Statistical significance of individual units was estimated using receiver operating characteristic (ROC) analysis, comparing binocular responses to the monocular responses for each unit (perfcurve.m in MATLAB). Specifically, we used the power at the drift rate of the stimulus based on the oscillatory PSD described above, or the F1 response. To assess significance, we ran a random (Monte Carlo) shuffle control in which we shuffled, with replacement, across both conditions, creating two surrogate conditions. We then calculated the ROC curve for these surrogate comparisons. Because we tested for both binocular suppression and binocular facilitation, we used a threshold quantile of 0.975 for $\alpha=0.05$. Specifically, if the area under the curve exceeded the 0.975 quantile of the shuffled distribution of AUC values, the unit was considered to show significant binocular modulation for that condition. One exception to this criterion was made and explictly noted, where units showing suppression at $\alpha=$ 0.2 (or AUC $>0.9$ of the shuffled distribution) were included to increase $N$. To determine the direction of the modulation (binocular suppression or facilitation), we compared the means of the binocular and monocular conditions. If the mean response was greater under binocular stimulation than monocular stimulation, we deemed the neuron to show binocular facilitation; under the converse condition we deemed the neuron to show binocular suppression. The contrast levels used across recording days was not perfectly consistent. To consolidate data for population averages, we grouped the following contrast ranges: $0.0,0.025-0.05$, $0.106-0.135,0.318-0.368,0.8-1.0$. These ranges were based on clustering of the contrast levels sampled across units. For visualization, data are presented at the median value of the relevant range.

\section{MRI}

MRI was used to aid electrode placement. Animals were anesthetized using the procedure outlined above then placed in a Philips Achieva 3T MRI scanner $(80 \mathrm{mT} / \mathrm{m}$ gradient strength, $200 \mathrm{~T} / \mathrm{m} / \mathrm{s}$ slew-rate; Koninklijke Philips N. V.) Vital signs were monitored continuously. T1-weighted 3D MPRAGE scans were acquired with a 32-channel head coil equipped for SENSE imaging. Images were acquired using a $0.5-\mathrm{mm}$ isotropic voxel resolution with the following parameters: repetition time (TR) $5 \mathrm{~s}$, echo time (TE) $2.5 \mathrm{~ms}$, flip angle $7^{\circ}$.

\section{Code accessibility}

Custom-written code used for this study is available from the corresponding author on request.

\section{Results}

Our primary goal was to determine how visual responses of LGN neurons in awake primates are 
influenced by the eye from which they do not receive direct retinal input (the non-dominant eye). In common with prior work in anesthetized animals (Tong et al., 1992; Sengpiel et al., 1995; Belluccini et al., 2019), we tested this question by varying the contrast of the stimulus presented to each eye, thereby adjusting the relative strength of the signal each eye carried. In the following sections, we first consider whether the presence of stimulus in the non-dominant eye impacts visual response to stimuli presented at different contrasts to the dominant eye. Then, we consider how binocular modulation is impacted by the contrast in the non-dominant eye.

One important feature of our experimental setup was that all stimuli were presented through a calibrated mirror stereoscope (Fig. 1; Materials and Methods). Thus, we were able to hold mean luminance constant across both eyes and avoided effects of short-term monocular deprivation that can arise if one eye is patched (Lunghi et al., 2015; Zhou et al., 2015; Binda et al., 2018; Wang et al., 2020). Under monocular conditions, the dominant eye of the recorded neurons was stimulated at either low (0.12), medium (0.34), or high (0.9) contrast levels. In pseudorandomized separate trials, the dominant eye viewed the same (low, medium, or high contrast) stimulus while the non-dominant eye was presented with either a high (0.9) or medium (0.34) contrast stimulus. In total we collected spiking data from $56 \mathrm{LGN}$ single units (41 units in monkey 134, 15 in monkey B52) across 40 sessions (27 sessions in 134, 13 in monkey B52). Note that while we used multicontact arrays, we were unable to record from multiple units simultaneously for most sessions. The reason for this limitation is that we entered the brain orthogonal to the cortex above the LGN, and because of the shape of the LGN, the receptive field locations across the probe did not typically align. The benefit of the multicontact array was that the multiunit hash on the contacts provided context of the location of the electrode in the brain.

Macaques tend to make fixational eye movements such as microsaccades as well as small shifts in initial fixation position from trial-to-trial. In this study, variability in initial fixation position from trial-to-trial was substantial enough to presumably cause LGN receptive fields to shift in spatial position with respect to the stimulus. This variance in initial fixation position, and therefore initial phase of the grating stimulus over the RF, could cause the LGN spiking responses exhibit eye movement-related shifts in phase, both across and within trials (Fig. 2A). For example, the range of the initial (mean position between 0 and $15 \mathrm{~ms}$ poststimulus onset) horizontal and vertical eye positions was $0.99^{\circ}$ and $1.4^{\circ}$, respectively, for a file from the session shown in Figure $2 A$. We obviated this problem by calculating the autocorrelation of spikes for every trial (Fig. 2B), followed by averaging to yield the phase-aligned linear response to the stimulus (a grating drifting at $4 \mathrm{~Hz}$; Fig. $2 \mathrm{C}$ ).

We first identified each LGN neuron as $\mathrm{P}, \mathrm{M}$, or K by analyzing their respective responses to stimuli of varying spectral content and contrast (see Materials and Methods; Lee et al., 1989b). Responses to different spectral (cone-isolating) stimuli are shown for example $\mathrm{P}, \mathrm{M}$ and $\mathrm{K}$ units in Figure 3. In all, we identified $20 \mathrm{P}, 30 \mathrm{M}$,

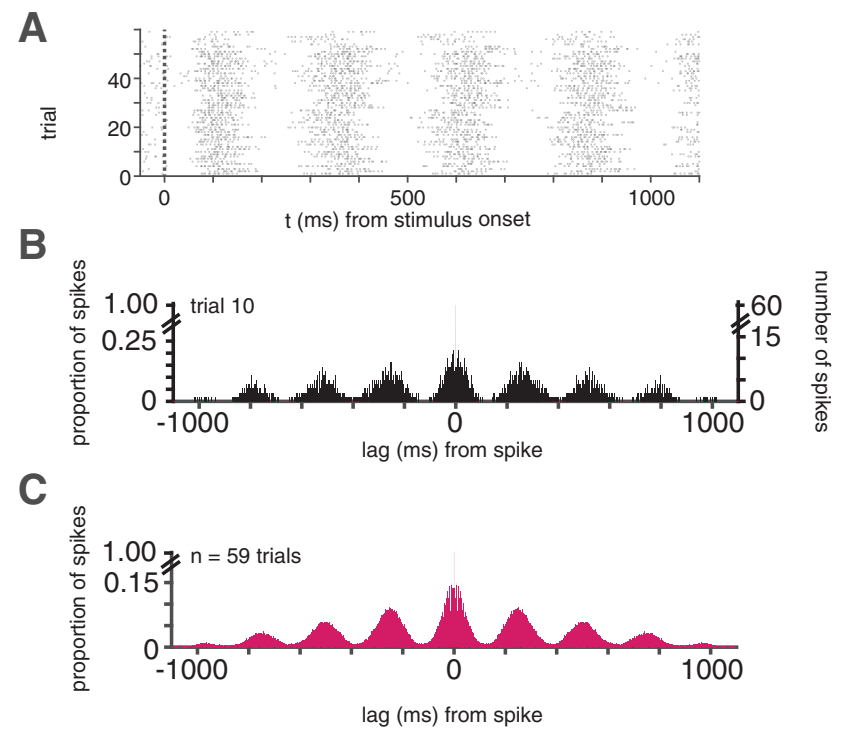

Figure 2. Schematic of autocorrelation-based analysis of LGN linear visual responses. $\boldsymbol{A}$, Note small changes in the onset of responses across trials likely because of small shifts in the animals' fixation position (see Materials and Methods). Calculation of autocorrelation of the spike train during the visual stimulation period for example trial. $\boldsymbol{B}$, Conversion of autocorrelation into units of spiking probability as function of lag for single trial. $\boldsymbol{C}$, Average spiking probabilities across trials.

and $6 \mathrm{~K}$ neurons based on their spectral responses (see Materials and Methods). The average CRFs of each of these groups matched expectations based on previous reports (Fig. 4; Shapley et al., 1981; Derrington and Lennie, 1984; Norton et al., 1988; White et al., 2001).

\section{Binocular modulation as a function of contrast in the dominant eye}

We first considered whether there was a difference between binocular stimulation and monocular stimulation of LGN neurons in general. To test for binocular modulation across the entire sample, we calculated the percent difference between monocular responses and binocular responses, with the same contrast in the dominant eye and contrast above 0 in the non-dominant eye. The median percent difference between monocular and binocular conditions was $-2.65 \%$, with no significant difference from 0 (Wilcoxon signed-rank, $p=0.11$ ) across the distribution. Therefore, there was no effect of binocular stimulation if contrast levels were not considered (Fig. 5A).

Next, we evaluated whether binocular modulation depends on contrast shown to the dominant eye. When we considered stimulation of the dominant eye at high, medium, and low contrast levels, a clear pattern emerged: non-dominant eye effects were present when the dominant eye was stimulated at low contrast (median: $-9.95 \%$; Wilcoxon sign-rank, $p=0.0024$; Fig. $5 B$ ) but not at medium (median: 2.58\%; Wilcoxon sign-rank, $p=0.35$ ) or high (median: $-0.73 \%$; Wilcoxon sign-rank, $p=0.77$ ) contrast levels. Based on the distribution and median of the sample, binocular modulation was predominantly 
A
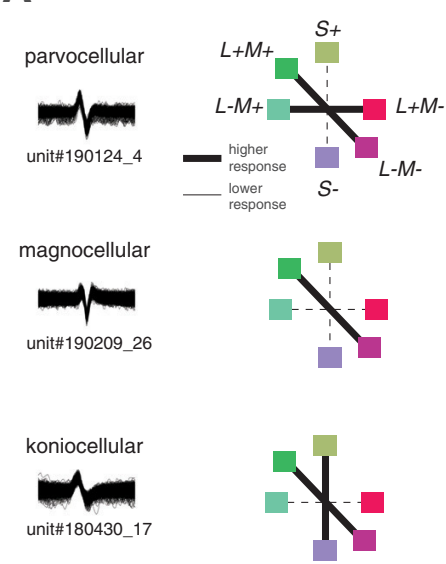

C
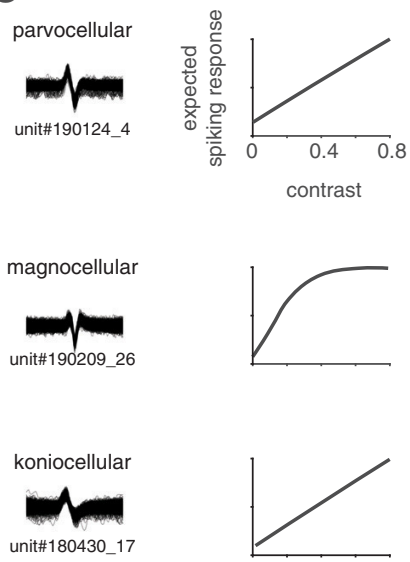

B
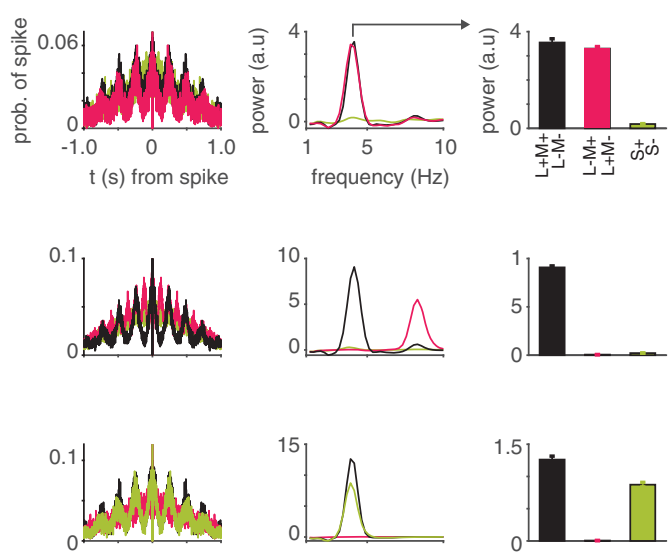

D
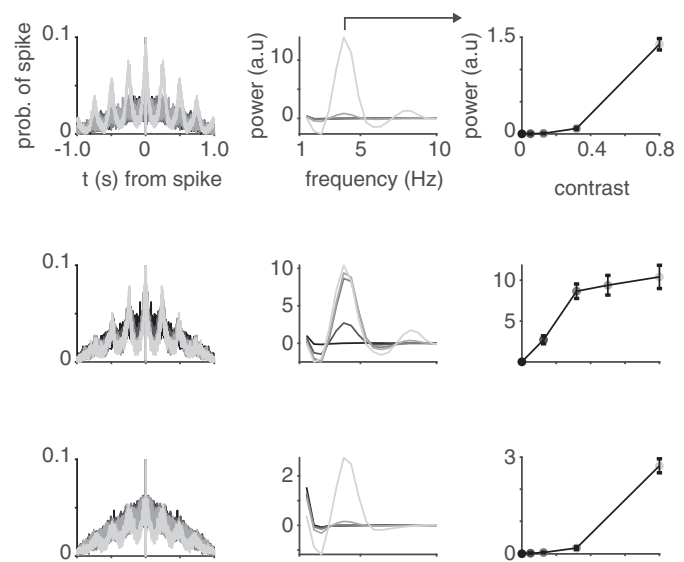

Figure 3. Neurophysiological characterization of $P, M$, and K LGN neurons. $\boldsymbol{A}$, Waveforms from individual spikes $(N>1000)$ for representative $P, M$, and $K$ units (left). Expected responses across $L^{+} M^{+} / L^{-} M^{-}, L^{-} M^{+} / L^{+} M^{-}$, and $S^{+} / S^{-}$stimulus conditions, expressed in DKL color space for each neuron type (bolder lines indicate stronger responses; right). $\boldsymbol{B}$, Average spike autocorrelation for $\mathrm{L}^{+} \mathrm{M}^{+} / \mathrm{L}^{-} \mathrm{M}^{-}, \mathrm{L}^{-} \mathrm{M}^{+} / \mathrm{L}^{+} \mathrm{M}^{-}$, and $\mathrm{S}^{+} / \mathrm{S}^{-}$stimulus conditions (left), average PSD on the autocorrelations (center), and the mean power of the PSD at the temporal frequency of the drifting grating (right) for each example P (top), M (middle), and K unit (bottom). Error bars indicate SEM. Power is the difference between the power of the signal and the estimated fractal power (see Materials and Methods). Note that the prominent $8-\mathrm{Hz}$ modulation for the $1 \mathrm{cycle} /{ }^{\circ} 4-\mathrm{Hz}$ drifting $\mathrm{L}^{+} \mathrm{M}^{-} / \mathrm{L}^{-} \mathrm{M}^{+}$stimulus results from $\mathrm{M}$ neurons receiving input from retinal ganglion cells that sum inputs from $L$ and $M$ cones (see Lee et al., 1989b). $\boldsymbol{C}$. The same units as in $\boldsymbol{A}$ with expected contrast response curve shapes based on published data cited in Results. $\boldsymbol{D}$, Same as in $\boldsymbol{B}$ but for different contrast levels, with each line representing a different contrast level, where darker colors represent lower contrast levels. Data within a range around each contrast point were averaged (see Materials and Methods).

suppressive. Therefore, stimulating the non-dominant eye tended to reduce spiking responses of LGN neurons when the dominant eye was stimulated at low contrast.

We next considered whether binocular modulation was specific to one or more LGN subclasses. To do so, we assessed whether there was a significant difference between monocular and binocular stimulation for each unit in our sample that we had identified as $\mathrm{P}, \mathrm{M}$, or $\mathrm{K}$ as described above. We found significant binocular modulation in units belonging to all three $(\mathrm{P}, \mathrm{M}$, and $\mathrm{K})$ subclasses (Fig. 5B). This analysis also revealed more instances of binocular suppression than facilitation at every contrast level in the dominant eye. There was no clear division across subclasses with respect to binocular modulation, except that none of the sampled $\mathrm{K}$ units showed significant modulation when high contrast was delivered through the dominant eye. These findings suggest that weak stimulation of the dominant eye allows for, predominantly suppressive, influences of the non-dominant eye across all major types of LGN neurons. This result motivated us to investigate more closely the effect of the nondominant eye.

\section{Influence of the non-dominant eye}

As a next step, we compared effects of a medium and high contrast stimulus delivered through the non-dominant eye. Doing so, we found that significant suppression occurred only when we presented a high contrast stimulus in the non-dominant eye and a low contrast stimulus in the dominant eye, though a medium contrast stimulus in the non-dominant eye trended toward significance (low 


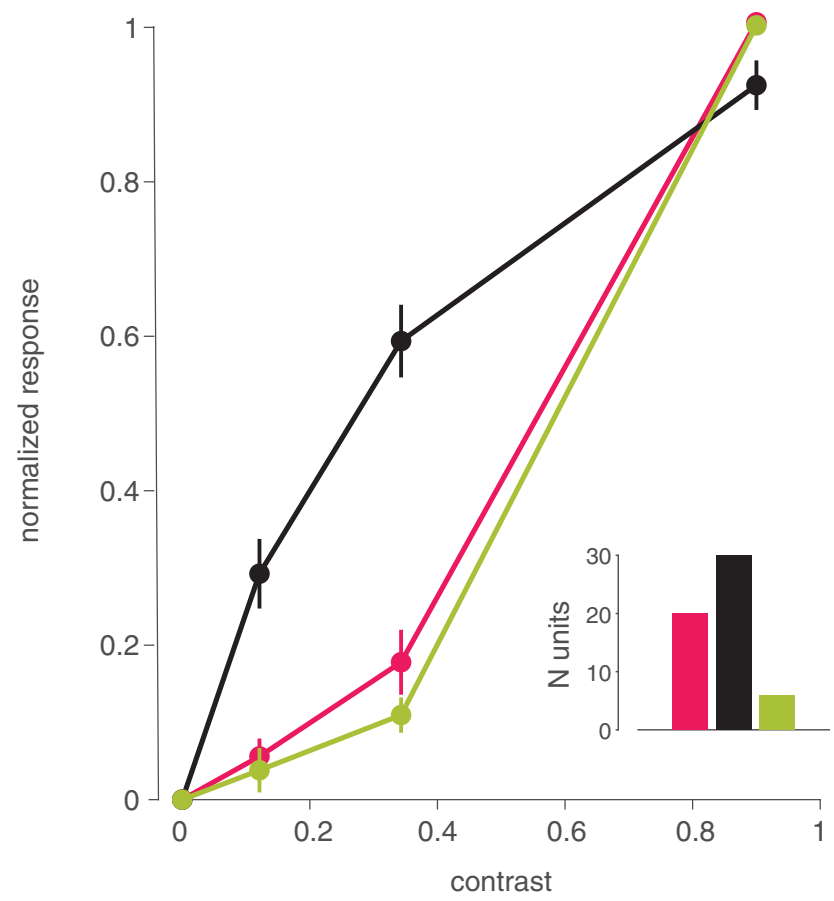

Figure 4. Mean CRFs for putative $P, M$, and $K$ neurons. Responses at different contrast levels were normalized between 0 and 1 for each unit, and averaged across units belonging to each group, with the maximum number of units in each group shown above. Error bars indicate SEM. Data within a contrast range around each contrast point listed were averaged (see Materials and Methods).

contrast: Wilcoxon sign-rank, $p=0.013$; medium contrast: Wilcoxon sign-rank, $p=0.058$; Fig. $6 A$ ). In other words, significant suppression across the sample was limited to instances where the suppressive drive from the non-dominant eye was strong and the excitatory drive from the dominant eye was low (and thus perhaps insufficient to override the suppressive influence).

If the non-dominant eye influences LGN responses of the dominant eye in a contrast-dependent fashion, we would expect a trend with respect to the fraction of LGN units that show significant suppression across the contrast range. We thus considered the percentage of units showing binocular suppression (ROC analysis, $\alpha=0.2$ ) as a function of contrast in the dominant eye (Fig. 6B). Using a high contrast stimulus in the non-dominant eye, we found a negative slope (linear regression, slope $=-7.26$, $p=0.16, \mathrm{df}=1$ ) with the most units showing suppression at low contrasts and fewest at the highest contrast. There was no clear trend with medium contrast in the non-dominant eye (linear regression, slope $=1.04, p=0.49$, df $=1$ ). One interpretation of this flat line is that it represents a noise floor, as in, weak stimulation of the non-dominant eye has no measurable impact.

Another way to assess whether the non-dominant eye influences responses of the dominant eye in a contrastdependent fashion is to study the binocular modulation of units showing significant suppression at low contrast in the dominant eye (and high contrast in the non-dominant eye). While there were only 6 units that showed significant modulation at this level, their median binocular modulation decreased gradually with increasing contrast in the dominant eye (Fig. 6C, purple line). Assessing binocular modulation with a high contrast stimulus in the nondominant eye across all units, there was a similar trend (Fig. 6C, black line). However, this trend did not reach significance after Bonferroni correction (low vs medium: Wilcoxon sign-rank, $p=0.067$; medium vs high: Wilcoxon sign-rank, $p=0.86$; low vs high: Wilcoxon sign-rank, $p=0.032$ ).

\section{Discussion}

As far as we are aware, this is the first study to investigate contrast-dependent binocular interactions in the LGN of awake macaques. The main finding from this study is that significant binocular suppression occurs across all major LGN cell classes when the non-dominant eye is stimulated at high contrast and the dominant eye is stimulated at low contrast. The predominant effect of this kind of imbalanced binocular stimulation was suppression of LGN visual responses. This result is congruent with previous work, and reminiscent of findings from psychophysical and modeling studies that suggest an antagonistic relationship between the two eyes at a monocular stage of visual processing (Blake, 1989; Ding and Sperling, 2006; Baker et al., 2007; Ding and Levi, 2014).

\section{Relationship to prior work}

One prior study of LGN units in anesthetized macaques reported binocular interactions for both $P(X)$ and $M(Y)$ neurons ( $K$ neurons were not distinguished; Marrocco and McClurkin, 1979). A different study tested for changes from baseline firing when a stimulus was presented to the non-dominant eye alone (non-dominant suppression) and reported binocular modulation only for $M(Y)$ neurons (Rodieck and Dreher, 1979). We did not test stimulation of the non-dominant eye alone in the present study. Our results agree with Marrocco and McClurkin (1979) in that we observed binocular modulation in both $P$ and $M$ neurons.

Binocularly-driven excitatory responses have been reported for $\sim 30 \%$ of $\mathrm{K}$ neurons in marmoset monkeys (Cheong et al., 2013; Zeater et al., 2015). In a more recent study, Belluccini et al. (2019) observed that most marmoset $\mathrm{K}$ neurons with excitatory responses to high-contrast stimuli showed binocular suppression. Consistent with this result, we observed only binocular suppression at low and medium contrasts in our relatively small sample of macaque $\mathrm{K}$ neurons.

The findings reported here are also in accordance with findings of binocular suppression and facilitation of LGN multiunit activity in awake macaques (Schroeder et al., 1990). These authors report $\sim 35-100 \%$ of sites show binocular suppression, with exact percentage depending on layer, except in layer P4 where no binocular suppression was observed. In the present study, the highest proportion of total neurons that showed binocular suppression was $\sim 25 \%$, which was observed using low contrast in the dominant eye. However, given the difference in underlying signals (population spiking vs single unit activity), it is 
A

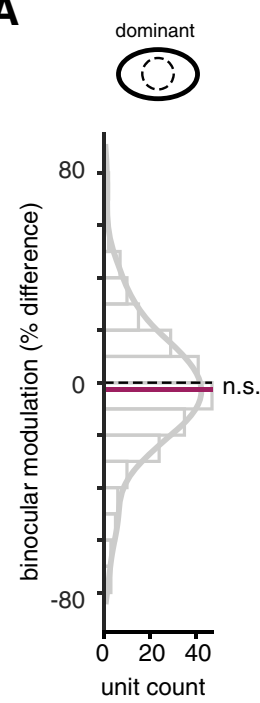

B
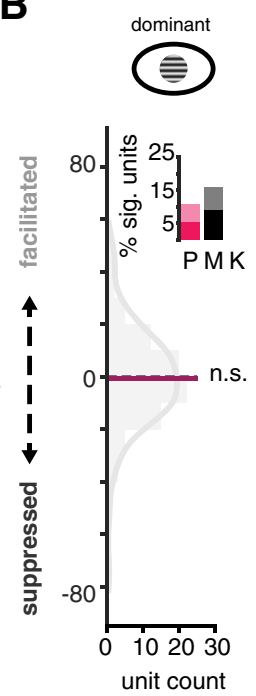
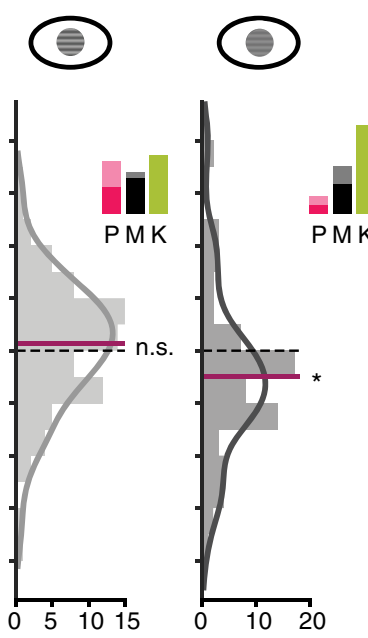

Figure 5. Binocular modulation occurs with low contrast in the dominant eye. A, Visual response difference between monocular and binocular stimulation across all contrast levels for all units. Percent difference calculated between monocular and binocular stimulation, with contrast matched in the dominant eye, shows no effect of stimulating the non-dominant eye. Black, horizontal dashed line demarcates division between binocular facilitation and suppression. Maroon, horizontal line marks the median of the distribution. Solid gray represents a non-parametric, kernel smoothing fit to the data. N.S. indicates binocular modulation across sample is not significantly different from 0 , alpha $=0.05$. B , Visual response difference between monocular and binocular stimulation for high (0.9), medium (0.34), and low (0.12) contrast gratings shown to the dominant eye, from left to right respectively. Bar graph insets show proportion of significant units (ROC analysis, $\alpha=0.05$ ) for each $\mathrm{P}, \mathrm{M}$, and $\mathrm{K}$ samples at respective contrast levels. Colors as in Figure 4 . Light shading of the bars corresponds to facilitated units. Asterisk indicates sample shows binocular modulation is significantly different from 0 , alpha $=0.05$.

difficult to compare these measures directly. Another consideration is that we compared monocular and binocular stimulation with drifting gratings of constant luminance that covered the RF, whereas Schroeder et al. (1990) used a high-intensity flash (strobe) stimulus.

In summary, this study and extant literature converge to the conclusion that binocular interactions in LGN is primarily suppressive in nature. This conclusion extends across anesthetized macaques and marmosets (Marrocco and McClurkin, 1979; Rodieck and Dreher, 1979; Belluccini et al., 2019) as well as cats (Sanderson et al., 1971; Schmielau and Singer, 1977; Rodieck and Dreher, 1979; Xue et al., 1987; Tong et al., 1992; Sengpiel et al., 1995) and to awake macaques (Schroeder et al., 1990; present study).

\section{Antagonistic relationship between the two eyes}

Across the sample, significant binocular modulation occurred only when there was a high contrast stimulus in the non-dominant eye and low contrast stimulus in the dominant eye. This result suggests that LGN neurons in awake primates are inhibited by the eye from which they receive no direct retinal input when that eye carries a signal much stronger than the eye from which they receive direct retinal input. Given that most of this modulation was suppressive, the pattern observed suggests a competitive relationship between the two eyes' signals at the earliest level of non-retinal visual processing.

The pattern of binocular modulation observed here is consistent with the phenomenon of interocular suppression more generally, in that more suppression occurs with greater contrast differences between the eyes (albeit only when that difference favors the non-dominant eye). The result reported in this study is also congruent with previous work in the cat that suggested interocular suppression occurs in about half of cat LGN neurons (Sengpiel et al., 1995).

Interocular suppression has been suggested to play a major role in binocular rivalry, the visual phenomenon resembling alternating views of each eye's perspective that arises when the images in the two eyes are too different to fuse (Blake, 1989). Note that in this study, we were exclusively focused on conditions that are known to induce binocular fusion: gratings of identical spatial position, orientation, spatial frequency and phase that only differed in interocular contrast. Interocular contrast differences alone are insufficient to induce binocular rivalry (Kertesz and Jones, 1970; O'Shea and Crassini, 1982; Ding and Sperling, 2006; Huang et al., 2010; Ding and Levi, 2017). Prior work in macaques suggest that LGN neurons do not alter their responses to rivalrous stimuli (Lehky and Maunsell, 1996), yet several human fMRI studies suggest otherwise (Haynes et al., 2005; Wunderlich et al., 2005). It thus would be interesting to revisit the role of different types of LGN neurons for binocular rivalry in future studies that incorporate perceptual report from the animal (Leopold et al., 2003).

The results presented here also call to mind models in which the two eyes share a mutually antagonistic, or competitive, relationship (Blake, 1989; Ding and Sperling, 2006; Baker et al., 2007; Ding and Levi, 2014): if the eyes compete at a monocular stage of processing, binocular interactions will be more likely to occur when disparate levels of contrast are presented to the two eyes. The non- 


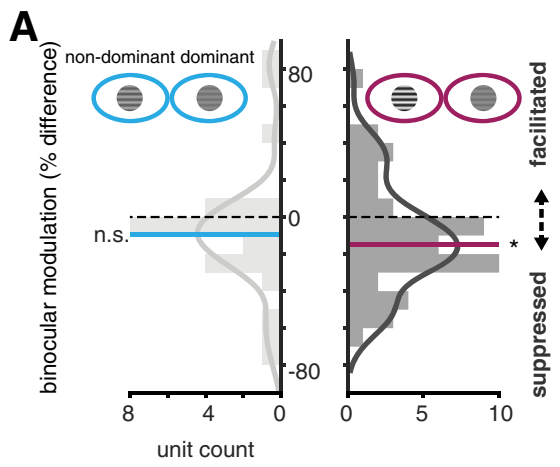

B
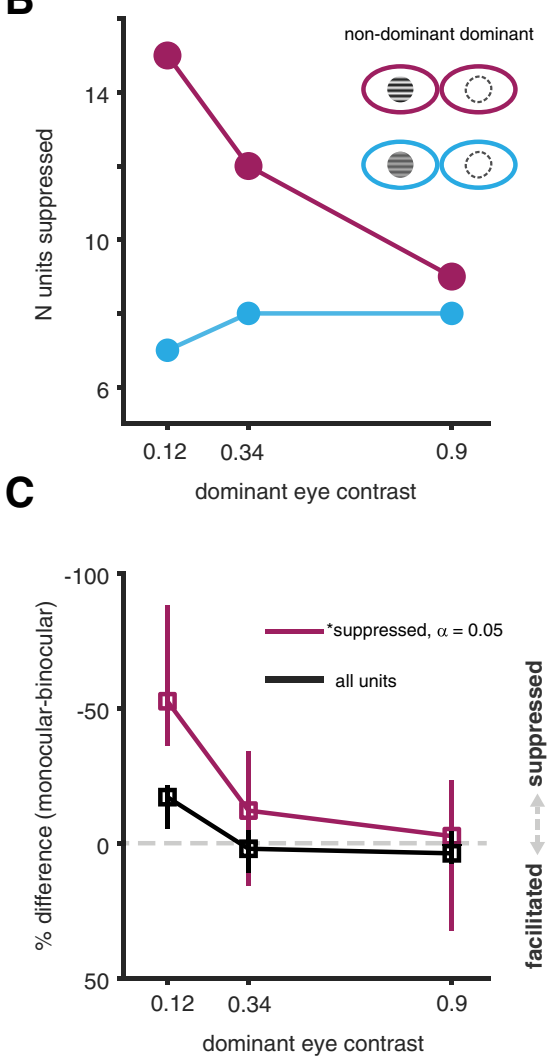

Figure 6. Comparison of binocular modulation at two different nondominant eye contrast levels. $\boldsymbol{A}$, left, Visual response difference between monocular and binocular stimulation with low (0.12) contrast in the dominant eye and medium (0.34) contrast in the non-dominant eye. Blue line marks the median of the distribution. Right, Same but with high (0.9) contrast in the non-dominant eye. Maroon line marks the median of the distribution. Other conventions as in Figure 5. B, Number of units that show suppression (ROC analysis, $\alpha=0.2$ ) across dominant eye contrast levels with medium (blue) and high (maroon) contrast in the non-dominant eye. $\boldsymbol{C}$, Median binocular modulation as a function of contrast in the dominant eye for units showing significant modulation (maroon, $\mathrm{ROC}, \alpha=0.05, N=6$ ) with high interocular contrast imbalance (low contrast dominant eye, high contrast non-dominant eye), as well as across the whole sample (black line). Error bars are 95\% confidence intervals on the medians.

dominant eye can reduce dominant eye responses to low contrast only if the contrast presented to the non-dominant eye is stronger than the contrast presented to the dominant eye. On the other hand, the neuron is less susceptible to the influence of the non-dominant eye if the contrast delivered through both eyes is more or less equal. Several models on binocular combination include interocular gain control at a monocular stage, before the two monocular streams are combined to a binocular signal (Truchard et al., 2000; Ding and Sperling, 2006; Meese et al., 2006; Baker et al., 2007). Indeed, the binocular suppression in the LGN we report in this study (see Figs. $5 B$, 6) qualitatively agrees with predictions for interocular gain control at a monocular stage. Note, however, that binocular suppression was statistically significant for the most extreme interocular contrast discrepancy only. While these results shed light on the functional architecture underlying how the two eyes interact at this early stage of visual processing, it is important to acknowledge the much more extensive binocular interactions that occur under broad stimulus conditions in primary visual cortex (Hubel and Wiesel, 1968; Smith et al., 1997; Moradi and Heeger, 2009). Thus, an alternative, albeit not mutually exclusive, explanation for binocular suppression in the LGN is that the suppression comes about through extraretinal circuits that mediate gain control in the LGN. For example, LGN contrast gain control has been shown to be impacted by cooling of visual cortical neurons (Przybyszewski et al., 2000). Gain control mechanisms in these or other binocular circuits could impact responses in LGN neurons through feedback projections.

As mentioned above, binocular suppression in the LGN was statistically significant only for the most extreme interocular contrast discrepancy, with higher contrast in the non-dominant eye. In individuals with normal binocular vision, contrast is often matched across the eyes. However, large interocular contrast differences might occur when one eye is closed, when one eye's view is obstructed (e. g., by the nose) or when the eyes are misaligned. Indeed, in individuals with strabismic amblyopia, mismatched contrasts across the eyes is necessary for equal performance on psychophysical tasks because of interocular suppression (Li et al., 2011). In any case, imbalanced contrast conditions created in the laboratory are informative in that they shed light on the nature of the (antagonistic) relationship between the two eyes at this early stage of visual processing.

\section{Neurophysiological mechanism for binocular modulation in the LGN}

Binocular modulation of LGN neurons in primates could arise, among other possibilities, through (1) local connections within the LGN, (2) connections between the TRN and LGN, or (3) connections from V1 to the LGN directly or via the TRN (for review, see Dougherty et al., 2019). Indeed, the source of binocular LGN modulation may be different for $\mathrm{P}, \mathrm{M}$, or $\mathrm{K}$ neurons, given the known specificity of corticogeniculate projections to the LGN (Briggs and Usrey, 2009). For K neurons specifically, binocular modulation could arise through connections between the superior colliculus and the LGN (Stepniewska et al., 1999; Zeater et al., 2019). Because of the variance in initial fixation position around the fixation cross (that presumably led to RF shifts with respect to the sinusoidal grating), we 
were unable to analyze the onset latency of modulation to infer the source of the modulation to distinguish between these possibilities. More work will be needed to determine the source of binocular modulation in the LGN.

In summary, the findings presented in this study build on previous experiments (Marrocco and McClurkin, 1979; Rodieck and Dreher, 1979; Schroeder et al., 1990; Belluccini et al., 2019) by showing that a minority of LGN neurons are sensitive to both eyes in awake primates. The pattern of modulation across contrast levels nevertheless suggests a role of LGN neurons in suppressing large interocular contrast differences.

\section{References}

Asaad WF, Santhanam N, McClellan S, Freedman DJ (2013) Highperformance execution of psychophysical tasks with complex visual stimuli in MATLAB. J Neurophysiol 109:249-260.

Baker DH, Meese TS, Summers RJ (2007) Psychophysical evidence for two routes to suppression before binocular summation of signals in human vision. Neuroscience 146:435-448.

Belluccini E, Zeater N, Pietersen A, Eiber C, Martin P (2019) Binocular summation in marmoset lateral geniculate nucleus. Vis Neurosci 36:E012.

Binda P, Kurzawski JW, Lunghi C, Biagi L, Tosetti M, Morrone MC (2018) Response to short -term deprivation of the human adult visual cortex measured with 7T BOLD. eLife 7:e40014.

Blake R (1989) A neural theory of binocular rivalry. Psychol Rev 96:145-167.

Briggs F, Usrey WM (2009) Parallel processing in the corticogeniculate pathway of the macaque monkey. Neuron 62:135-146.

Campos-Ortega JA, Glees P, Neuhoff V (1968) Ultrastructural analysis of individual layers in the lateral geniculate body of the monkey. Z Zellforsch Mikrosk Anat 87:82-100.

Cheong SK, Tailby C, Solomon SG, Martin PR (2013) Cortical-like receptive fields in the lateral geniculate nucleus of marmoset monkeys. J Neurosci 33:6864-6876.

Derrington AM, Lennie P (1984) Spatial and temporal contrast sensitivities of neurones in lateral geniculate nucleus of macaque. J Physiol 357:219-240.

Derrington AM, Krauskopf J, Lennie P (1984) Chromatic mechanisms in lateral geniculate nucleus of macaque. J Physiol 357:241-265.

Ding J, Sperling G (2006) A gain-control theory of binocular combination. Proc Natl Acad Sci USA 103:1141-1146.

Ding J, Levi DM (2014) Rebalancing binocular vision in amblyopia. Ophthalmic Physiol Opt 34:199-213.

Ding J, Levi DM (2017) Binocular combination of luminance profiles. $\mathrm{J}$ Vis 17:4.

Dougherty K, Schmid MC, Maier A (2019) Binocular response modulation in the lateral geniculate nucleus. J Comp Neurol 527:522534.

Hanes DP, Thompson KG, Schall JD (1995) Relationship of presaccadic activity in frontal eye field and supplementary eye field to saccade initiation in macaque: Poisson spike train analysis. Exp Brain Res 103:85-96.

Haynes JD, Deichmann R, Rees G (2005) Eye-specific effects of binocular rivalry in the human lateral geniculate nucleus. Nature 438:496-499.

Huang CB, Zhou J, Zhou Y, Lu ZL (2010) Contrast and phase combination in binocular vision. PLoS One 5:e15075.

Hubel DH, Wiesel TN (1968) Receptive fields and functional architecture of monkey striate cortex. J Physiol 195:215-243.

Kaas JH, Guillery RW, Allman JM (1972) Some principles of organization in the dorsal lateral geniculate nucleus. Brain Behav Evol 6:253-299.

Kertesz AE, Jones RW (1970) Human cyclofusional response. Vision Res 10:891-896.
Lamb TD (1995) Photoreceptor spectral sensitivities: common shape in the long-wavelength region. Vision Res 35:3083-3091.

Lee BB, Martin PR, Valberg A (1989a) Sensitivity of macaque retinal ganglion cells to chromatic and luminance flicker. J Physiol 414:223-243.

Lee BB, Martin PR, Valberg A (1989b) Nonlinear summation of Mand L-cone inputs to phasic retinal ganglion cells of the macaque. J Neurosci 9:1433-1442.

Lehky SR, Maunsell JH (1996) No binocular rivalry in the LGN of alert macaque monkeys. Vision Res 36:1225-1234.

Leopold DA, Maier A, Logothetis NK (2003) Measuring subjective visual perception in the nonhuman primate. J Conscious Stud 10:115-130.

Li J, Thompson B, Lam CSY, Deng D, Chan LYL, Maehara G, Woo GC, Yu M, Hess RF (2011) The role of suppression in amblyopia. Invest Ophthalmol Vis Sci 52:4169-4176.

Lund JS, Lund RD, Hendrickson AE, Bunt AH, Fuchs AF (1975) The origin of efferent pathways from the primary visual cortex, area 17, of the macaque monkey as shown by retrograde transport of horseradish peroxidase. J Comp Neurol 164:287-303.

Lunghi C, Berchicci M, Morrone MC, Di Russo F (2015) Short-term monocular deprivation alters early components of visual evoked potentials. J Physiol 593:4361-4372.

Marrocco RT, McClurkin JW (1979) Binocular interaction in the lateral geniculate nucleus of the monkey. Brain Res 168:633-637.

Meese TS, Georgeson MA, Baker DH (2006) Binocular contrast vision at and above threshold. J Vis 6:1224-1243.

Moradi F, Heeger DH (2009) Inter-ocular contrast normalization in human visual cortex. J Vis 9:13.1-22.

Norton TT, Casagrande VA, Irvin GE, Sesma MA, Petry HM (1988) Contrast-sensitivity functions of $\mathrm{W}-, \mathrm{X}$-, and $\mathrm{Y}$-like relay cells in the lateral geniculate nucleus of bush baby, Galago crassicaudatus. J Neurophysiol 59:1639-1656.

O'Shea RP, Crassini B (1982) The dependence of cyclofusion on orientation. Percept Psychophys 32:195-196.

Pachitariu M, Steinmetz N, Kadir S, Carandini M, Harris KD (2016) Kilosort: realtime spike-sorting for extracellular electrophysiology with hundreds of channels. bioRxiv. doi: https://doi.org/10.1101/ 061481.

Przybyszewski AW, Gaska JP, Foote W, Pollen DA (2000) Striate cortex increases contrast gain of macaque LGN neurons. Vis Neurosci 17:485-494.

Rodieck RW, Dreher B (1979) Visual suppression from nondominant eye in the lateral geniculate nucleus: a comparison of cat and monkey. Exp Brain Res 35:465-477.

Saini KD, Garey LJ (1981) Morphology of neurons in the lateral geniculate nucleus of the monkey. Exp Brain Res 42:235-248.

Sanderson KJ, Bishop PO, Darian-Smith I (1971) The properties of the binocular receptive fields of lateral geniculate neurons. Exp Brain Res 13:178-207.

Sayer RJ, Friedlander MJ, Redman SJ (1990) The time course and amplitude of EPSPs evoked at synapses between pairs of CA3/ CA1 neurons in the hippocampal slice. J Neurosci 10:826-836.

Schmielau F, Singer W (1977) The role of visual cortex for binocular interactions in the cat lateral geniculate nucleus. Brain Res 120:354-361.

Schroeder CE, Salinger WL, Guido W (1988) The influence of anesthesia upon binocular processes controlling the recordability of $X$ and $\mathrm{Y}$-cells in the lateral geniculate nucleus of the cat. Brain Res 454:227-237.

Schroeder CE, Tenke CE, Arezzo JC, Vaughan HG (1990) Binocularity in the lateral geniculate nucleus of the alert macaque. Brain Res 521:303-310.

Sclar G, Maunsell JH, Lennie P (1990) Coding of image contrast in central visual pathways of the macaque monkey. Vision Res 30:110.

Sengpiel F, Blakemore C, Harrad R (1995) Interocular suppression in the primary visual cortex: a possible neural basis of binocular rivalry. Vision Res 35:179-195. 
Shapley R, Kaplan E, Soodak R (1981) Spatial summation and contrast sensitivity of $X$ and $Y$ cells in the lateral geniculate nucleus of the macaque. Nature 292:543-545.

Smith IIE, Chino Y, Ni J, Cheng H (1997) Binocular combination of contrast signals by striate cortical neurons in the monkey. $J$ Neurophysiol 78:366-382.

Stepniewska I, Qi HX, Kaas JH (1999) Do superior colliculus projection zones in the inferior pulvinar project to MT in primates? Eur $\mathrm{J}$ Neurosci 11:469-480.

Tong L, Guido W, Tumosa N, Spear PD, Heidenreich S (1992) Binocular interactions in the cat's dorsal lateral geniculate nucleus, II: effects on dominant-eye spatial-frequency and contrast processing. Vis Neurosci 8:557-566.

Truchard AM, Ohzawa I, Freeman RD (2000) Contrast gain control in the visual cortex: monocular versus binocular mechanisms. J Neurosci 20:3017-3032.

Wang M, McGraw P, Ledgeway T (2020) Short-term monocular deprivation reduces inter-ocular suppression of the deprived eye. Vision Res 173:29-40.

Wen H, Liu Z (2016) Separating fractal and oscillatory components in the power spectrum of neurophysiological signal. Brain Topogr 29:13-26.

White AJ, Wilder HD, Goodchild AK, Sefton AJ, Martin PR (1998) Segregation of receptive field properties in the lateral geniculate nucleus of a New-World monkey, the marmoset Callithrix jacchus. J Neurophysiol 80:2063-2076.

White AJ, Solomon SG, Martin PR (2001) Spatial properties of koniocellular cells in the lateral geniculate nucleus of the marmoset Callithrix jacchus. J Physiol 533:519-535.

Wiesel TN, Hubel DH (1966) Spatial and chromatic interactions in the lateral geniculate body of the rhesus monkey. J Neurophysiol 29:1115-1156.

Wunderlich K, Schneider KA, Kastner S (2005) Neural correlates of binocular rivalry in the human lateral geniculate nucleus. Nat Neurosci 8:1595-1602.

Xue JT, Ramoa AS, Carney T, Freeman RD (1987) Binocular interaction in the dorsal lateral geniculate nucleus of the cat. Exp Brain Res 68:305-310.

Zeater N, Cheong SK, Solomon SG, Dreher B, Martin PR (2015) Binocular visual responses in the primate lateral geniculate nucleus. Curr Biol 25:3190-3195.

Zeater N, Buzás P, Dreher B, Grünert U, Martin PR (2019) Projections of three subcortical visual centers to marmoset lateral geniculate nucleus. J Comp Neurol 527:535-545.

Zhou J, Baker HB, Simard M, Saint-Amour D, Hess RF (2015) Shortterm monocular patching boosts the patched eye's response in visual cortex. Restor Neurol Neurosci 33:381-387. 